\title{
Magnetic Oldroydian Viscoelastic Fluid - Analysis of Vorticity Transport
}

\author{
Pardeep Kumar $^{1 *}$--- Hari Mohan ${ }^{2}$ \\ ${ }^{1,2}$ Department of Mathematics, ICDEOL, Himachal Pradesh University, Shimla-171005, India. \\ ${ }^{1}$ Email:pldureja@gmail.com
}

\begin{abstract}
The transport of vorticity in Oldroydian viscoelastic fluid in the presence of suspended magnetic particles is considered. Equations governing the transport of vorticity in Oldroydian viscoelastic fluid in the presence of suspended magnetic particles are obtained from the equations of magnetic fluid flow proposed by Wagh and Jawandhia in their 1996 study on the transport of vorticity in magnetic fluid. From these equations, it follows that the transport of solid vorticity is coupled with the transport of fluid vorticity . Further, it is found that due to thermo-kinetic process, fluid vorticity may exist in the absence of solid vorticity, but when fluid vorticity is zero, then solid vorticity is necessarily zero. A two-dimensional case is also studied.
\end{abstract}

Keywords: Oldroydian viscoelastic Fluid, Suspended magnetic particles, Vorticity.

Licensed: This work is licensed under a Creative Commons Attribution 4.0 License.

\section{Introduction}

Magnetic fluids are those fluids in which magnetic particles are suspended in a liquid carrier. It is, thus, a two-phase system, consisting of solid and liquid phases. We assume that the liquid phase is non-magnetic in nature and magnetic force acts only on the magnetic particles; changing their velocity. Consequently, the dragging force acting on the carrier liquid is changed and thus the flow of carrier liquid is also influenced by the magnetic force. Because of the relative velocity between the solid and liquid particles, the net effect of the particles suspended in the fluid is extra dragging force acting on the system. Taking this force into consideration, Saffman [2] proposed the equations of the flow of suspension of non-magnetic particles. These equations were modified by Wagh [3] to describe the flow of magnetic fluid, by including the magnetic body force. Wagh and Jawandhia [1] have studied the transport of vorticity in a magnetic fluid. Transport and sedimentation of suspended particles in inertial pressure-driven flow has been considered by Yan and Koplik [4].

In all the above studies, the fluid has been considered to be as Newtonian, but many industrially important fluids (molten plastics, polymers, pulps and foods) exhibit a non-Newtonian fluid behaviour. Many common materials (paints and plastics) and more exotic ones (silicic magma, saturated soils, and the Earth's lithosphere) behave as viscoelastic fluids. With the growing importance of non-Newtonian fluids in modern technology and industries, investigations on such fluids are desirable. Widely used theoretical models (models A and B, respectively) for certain classes of viscoelastic fluids have been proposed by Oldroyd [5]. An experimental demonstration by Toms and Strawbridge [6] has revealed that a dilute solution of methyl methacrylate in n-butyl acetate agrees well with the theoretical model of Oldroyd [5]. The nature of the instability and other factors can affect viscoelastic fluids differently than Newtonian fluids. For example, the 
thermal instability of Maxwellian viscoelastic fluid in the presence of a uniform rotation has been considered by Bhatia and Steiner [7] where rotation is found to have a destabilizing effect, which is in contrast to the thermal instability of a Newtonian fluid where rotation has a stabilizing effect. The thermal instability of an Oldroydian viscoelastic fluid acted on by a uniform rotation has been studied by Sharma [8] and it was found that the rotation has both a destabilizing and, under certain conditions, a stabilizing effect in contrast to a Maxwell viscoelastic fluid, where it has a destabilizing effect. Hamabata and Namikawa [9] studied the propagation of thermoconvective waves in the Oldroyd fluid. Kumar et al. [10] considered the instability of the plane interface between two Oldroydian viscoelastic superposed fluids in the presence of uniform rotation and variable magnetic field in a porous medium. The problem of the onset of electro-hydrodynamic instability in a horizontal layer of Oldroydian viscoelastic dielectric liquid through Brinkman porous medium under the simultaneous action of a vertical a.c. electric field and a vertical temperature gradient is analyzed by El-Sayed [11]. Hoshoudy [12] has considered the quantum effects on the Rayleigh-Taylor instability of viscoelastic plasma model through a porous medium. Kumar and Mohan [13] have mathematically investigated the double-diffusive convection in an Oldroydian viscoelastic fluid under the simultaneous effects of magnetic field and suspended particles through porous medium. In another study, Kumar and Mohan [14] have considered the instability of an Oldroydian heterogeneous viscoelastic fluid in a porous medium. The boundary-value problem for the steady isothermal flow of an incompressible viscoelastic liquid of Oldroyd type in a bounded domain with a Navier type slip boundary condition has been considered by Le Roux [15]. El-Sayed et al. [16] have considered the effects of a vertical a.c. electric field and heat transfer on a peristaltic flow of an incompressible dielectric viscoelastic fluid in asymmetric flexible channel. An incompressible Oldroydian viscoelastic fluid layer heated and soluted from below in the presence of suspended (dust) particles and uniform vertical magnetic field to include the effect of Hall currents in porous medium has been considered by Kumar and Kumar [17]. The triply diffusive convection in an Oldroydian viscoelastic fluid is mathematically investigated in the absence and presence of magnetic field through porous medium by Kumar et al. [18].

Keeping in mind the importance of non-Newtonian fluids in modern technology and industries, we here attempt to study the transport of vorticity in magnetic Oldroydian viscoelastic fluid-particle mixtures.

\section{Basic Assumptions and Magnetic Body Force}

Particles of magnetic material are much larger than the size of the molecules of carrier liquid. Accordingly, we consider the limit of a microscopic volume element in which a fluid can be assumed to be a continuous medium and the magnetic particles must be treated as discrete entities. Now, if we consider a cell of magnetic fluid containing a larger number of magnetic particles, then we must consider the microrotation of the cell in addition to its translations as a point mass. Thus, one has to assign average velocity $\vec{q}_{d}$ and the average angular velocity $\vec{\omega}$ to the cell. But, here as an approximation, we neglect the effect of microrotation. We shall also make the following assumptions:

(i) Most of the ferrofluids are relatively poor conductors and hence free current density $\vec{J}$ is negligible and hence $\vec{J} \times \vec{B}$ is assumed to be insignificant.

(ii) The magnetic field is assumed to be curl free i.e. $\nabla \times \vec{H}=0$.

(iii) The liquid compressibility is unimportant in many situations. Hence contribution due to magnetic friction can be neglected. The remaining force of magnetic field is called as magnetization force.

(iv) All time-dependent magnetization effects in the fluid (such as hysteresis) are assumed to be negligible and the magnetization $\vec{M}$ is assumed to be collinear with $\vec{H}$.

From electromagnetic theory, the force per unit volume (in MKS units) on a piece of magnetized material of magnetization $\vec{M}$ (i.e. dipole moment per unit volume) in the field of magnetic intensity $\vec{H}$ is $\mu_{0}(\vec{M} . \nabla) \vec{H}$, where $\mu_{0}$ is the free-space permeability. Using assumption (iv), we obtain

$$
\mu_{0}(\vec{M} . \nabla) \vec{H}=\frac{\mu_{0} M}{H}(\vec{H} . \nabla) \vec{H}, \text { where } M=|\vec{M}| \text { and } H=|\vec{H}| .
$$

But by assumption (ii), we have

$$
\begin{aligned}
(\vec{H} . \nabla) \vec{H}=\frac{1}{2} \nabla(\vec{H} \cdot \vec{H})-\vec{H} \times(\nabla \times \vec{H})=\frac{1}{2} \nabla(\vec{H} \cdot \vec{H}) . \\
\text { Hence } \mu_{0}(\vec{M} \cdot \nabla) \vec{H}=\left(\frac{\mu_{0} M}{H}\right) \frac{1}{2} \nabla(\vec{H} \cdot \vec{H})=\mu_{0} M \nabla H .
\end{aligned}
$$

The magnetic body force therefore assumes the form [Rosensweig [19]] 


$$
f_{m}=\mu_{0} M \nabla H .
$$

\section{Derivation of Equations Governing Transport of Vorticity in Magnetic Oldroydian Viscoelastic Fluid}

Let $\Gamma_{i j}, \tau_{i j}, e_{i j}, \mu, \lambda, \lambda_{0}(<\lambda), p, \delta_{i j}, q_{i}, x_{i}$ and $d / d t$ denote respectively the total stress tensor, the shear stress tensor, the rate-of-strain tensor, the viscosity, the stress relaxation time, the strain retardation time, the isotropic pressure, the Kroneckor delta, the velocity vector, the position vector and the convective derivative. Then the Oldroydian viscoelastic fluid is described by the constitutive relations

$$
\begin{aligned}
& \Gamma_{i j}=-p \delta_{i_{j}}+\tau_{i j}, \\
& \left(1+\lambda \frac{d}{d t}\right) \tau_{i j}=2 \mu\left(1+\lambda_{0} \frac{d}{d t}\right) e_{i j}, \\
& e_{i j}=\frac{1}{2}\left(\frac{\partial q_{i}}{\partial x_{j}}+\frac{\partial q_{j}}{\partial x_{i}}\right),
\end{aligned}
$$

Relations of the type (4) were first studied by Oldroyd [5]. Oldroyd [5] also showed that many rheological equations of state; of general validity, reduce to (4) when linearized. $\lambda_{0}=0$ yields the fluid to be Maxwellian whereas $\lambda=\lambda_{0}=0$ gives the Newtonian viscous fluid.

Wagh [3] modified the Saffman's equations for flow of suspension to describe the flow of magnetic fluid by including the body force $\mu_{0} M \nabla H$ acting on the suspended magnetic particles. Now the equations expressing the flow of suspended magnetic particles and the flow of Oldroydian viscoelastic fluid in which magnetic particles are suspended are therefore written as

$$
\begin{aligned}
& m N\left[\frac{\partial \vec{q}_{d}}{\partial t}+\left(\vec{q}_{d} \cdot \nabla\right) \vec{q}_{d}\right]=m N \vec{g}+\mu_{0} M \nabla H+K N\left(\vec{q}-\vec{q}_{d}\right), \\
& \left(1+\lambda \frac{\partial}{\partial t}\right) \rho\left[\frac{\partial \vec{q}}{\partial t}+(\vec{q} \cdot \nabla) \vec{q}\right]=\left(1+\lambda \frac{\partial}{\partial t}\right)\left[-\nabla P+\rho \vec{g}+K N\left(\vec{q}_{d}-\vec{q}\right)\right]+\mu\left(1+\lambda_{0} \frac{\partial}{\partial t}\right) \nabla^{2} \vec{q},
\end{aligned}
$$

where $P, \rho, \mu, \vec{q}(u, v, w), \vec{g}(0,0,-g) ; \vec{q}_{d}(l, r, s), m, N(\bar{x}, t)$ denote, respectively, the pressure less the hydrostatic pressure, density, viscosity, velocity of the pure fluid, gravity force; velocity, mass and number density of the particles; $\bar{x}=(x, y, z), K=6 \pi \mu \eta, \eta$ being the particle radius, is the Stokes' drag coefficient and $m N$ is the mass of particles per unit volume.

If we assume that the particle has a uniform spherical shape and that the particle velocity relative to the fluid is small, then in the equations of motion for the fluid, because of the presence of suspended particles, an additional force term appears proportional to the velocity difference between the suspended particles and the fluid. Since the force exerted by the fluid on the suspended particles is equal and opposite to that exerted by the particles on the fluid, there must be an extra force term, equal in magnitude but opposite in sign appears in the equations of motion of the suspended particles. The buoyancy force on the particles is also neglected. This force is proportional to the quotient of $\rho$ and the particle density, and an analysis for the case of freefree boundary conditions shows that its small stabilizing effect is negligible. We assume that the distances between particles are quite large compared with their diameter, so interparticle reactions are also ignored. Making use of the Lagrange's vector identities

$$
\left(\vec{q}_{d} \cdot \nabla\right) \vec{q}_{d}=\frac{1}{2} \nabla q_{d}^{2}-\vec{q}_{d} \times \vec{\Omega},(\vec{q} \cdot \nabla) \vec{q}=\frac{1}{2} \nabla q^{2}-\vec{q} \times \vec{\Omega}_{1},
$$

Equations 5 and 6 become

$$
m N\left[\frac{\partial \vec{q}_{d}}{\partial t}-\left(\vec{q}_{d} \times \vec{\Omega}\right)\right]=-m N g z-m N \nabla q_{d}^{2}+\mu_{0} M \nabla H+K N\left(\vec{q}-\vec{q}_{d}\right),
$$




$$
\begin{aligned}
& \rho\left(1+\lambda \frac{\partial}{\partial t}\right)\left[\frac{\partial \vec{q}}{\partial t}-\left(\vec{q} \times \vec{\Omega}_{1}\right)\right]=\left(1+\lambda \frac{\partial}{\partial t}\right)\left[-\nabla P-\nabla \rho g z-\frac{1}{2} \rho \nabla q^{2}+K N\left(\vec{q}_{d}-\vec{q}\right)\right] \\
& +\mu\left(1+\lambda_{0} \frac{\partial}{\partial t}\right) \nabla^{2} \vec{q},
\end{aligned}
$$

where $\vec{\Omega}=\nabla \times \vec{q}_{d}$ and $\vec{\Omega}_{1}=\nabla \times \vec{q}$ are respectively the solid vorticity and fluid vorticity.

Taking the curl of these equations and recalling that the curl of a gradient is identically equal to zero, we get

$$
\begin{aligned}
& m N\left[\frac{\partial \vec{\Omega}}{\partial t}-\left(\nabla \times \vec{q}_{d} \times \vec{\Omega}\right)\right]=\mu_{0} \nabla \times M \nabla H+K N\left(\vec{\Omega}_{1}-\vec{\Omega}\right), \\
& \rho\left(1+\lambda \frac{\partial}{\partial t}\right)\left[\frac{\partial \vec{\Omega}_{1}}{\partial t}-\left(\nabla \times \vec{q} \times \vec{\Omega}_{1}\right)\right]=\mu\left(1+\lambda_{0} \frac{\partial}{\partial t}\right) \nabla^{2} \vec{\Omega}_{1}+\left(1+\lambda \frac{\partial}{\partial t}\right) K N\left(\vec{\Omega}-\vec{\Omega}_{1}\right) .
\end{aligned}
$$

By making use of the vector identities

$$
\begin{aligned}
& \nabla \times\left(\vec{q}_{d} \times \vec{\Omega}\right)=(\vec{\Omega} \cdot \nabla) \vec{q}_{d}-\left(\vec{q}_{d} \cdot \nabla\right) \vec{\Omega}+\vec{q}_{d} \nabla \cdot \vec{\Omega}-\vec{\Omega} \nabla \cdot \vec{q}_{d}=(\vec{\Omega} \cdot \nabla) \vec{q}_{d}-\left(\vec{q}_{d} \cdot \nabla\right) \vec{\Omega}, \\
& \nabla \times\left(\vec{q} \times \vec{\Omega}_{1}\right)=\left(\vec{\Omega}_{1} \cdot \nabla\right) \vec{q}-(\vec{q} \cdot \nabla) \vec{\Omega}_{1}+\vec{q} \nabla \cdot \vec{\Omega}_{1}-\vec{\Omega}_{1} \nabla \cdot \vec{q}=\left(\vec{\Omega}_{1} \cdot \nabla\right) \vec{q}-(\vec{q} \cdot \nabla) \vec{\Omega}_{1},
\end{aligned}
$$

Equations 10 and 11 become

$$
\begin{aligned}
& m N \frac{D \vec{\Omega}}{D t}=\mu_{0} \nabla \times M \nabla H+m N(\vec{\Omega} \cdot \nabla) \vec{q}_{d}+K N\left(\vec{\Omega}_{1}-\vec{\Omega}\right) \\
& \left(1+\lambda \frac{\partial}{\partial t}\right) \frac{D \vec{\Omega}_{1}}{D t}=v\left(1+\lambda_{0} \frac{\partial}{\partial t}\right) \nabla^{2} \vec{\Omega}_{1}+\left(1+\lambda \frac{\partial}{\partial t}\right)\left(\vec{\Omega}_{1} \cdot \nabla\right) \vec{q}+\left(1+\lambda \frac{\partial}{\partial t}\right) \frac{K N}{\rho}\left(\vec{\Omega}-\vec{\Omega}_{1}\right)
\end{aligned}
$$

where $v$ is the kinematic viscosity and $\frac{D}{D t}=\frac{\partial}{\partial t}+\left(\vec{q}_{d}, \nabla\right)$ is the convective derivative.

In Equation 14,

$$
\nabla \times(M \nabla H)=(\nabla M \times \nabla H)+(M \nabla \times \nabla H) \text {. }
$$

Since the curl of the gradient is zero, the last term in Equation 16 is zero. Also since

$$
M=M(H, T) \text {. We have }
$$

$$
\nabla M=\left(\frac{\partial M}{\partial H}\right) \nabla H+\left(\frac{\partial M}{\partial T}\right) \nabla T .
$$

By making use of (17), Equation 16 becomes

$$
\nabla \times(M \nabla H)=\left(\frac{\partial M}{\partial H}\right) \nabla H \times \nabla H+\left(\frac{\partial M}{\partial T}\right) \nabla T \times \nabla H .
$$

The first term on the right hand side of this equation is clearly zero, hence we get

$$
\nabla \times(M \nabla H)=\left(\frac{\partial M}{\partial T}\right) \nabla T \times \nabla H .
$$

Substituting this expression in Equation 14, we get

$$
m N \frac{D \vec{\Omega}}{D t}=\mu_{0}\left(\frac{\partial M}{\partial T}\right) \nabla T \times \nabla H+m N(\vec{\Omega} . \nabla) \vec{q}_{d}+K N\left(\vec{\Omega}_{1}-\vec{\Omega}\right) .
$$

Here (15) and (20) are the equations governing the transport of vorticity in magnetic Oldrodian viscoelastic fluid-particle mixtures.

In Equation 20 the first term in the right hand side $\mu_{0}\left(\frac{\partial M}{\partial T}\right) \nabla T \times \nabla H$ describes the production of vorticity due to thermo-kinetic processes. The last term $K N\left(\vec{\Omega}_{1}-\vec{\Omega}\right)$. gives the change in solid vorticity on account of exchange of vorticity between the liquid and solid.

From Equations 15 and 20, it follows that the transport of solid vorticity $\vec{\Omega}$ is coupled with the transport of fluid vorticity $\vec{\Omega}_{1}$. 
From Equation 20, we see that if solid vorticity $\vec{\Omega}$ is zero, then the fluid vorticity $\vec{\Omega}_{1}$ is non-zero and it is given by

$$
\vec{\Omega}_{1}=-\frac{\mu_{0}}{K N}\left(\frac{\partial M}{\partial T}\right) \nabla T \times \nabla H .
$$

This implies that due to thermo-kinetic processes, fluid vorticity may exist in the absence of solid vorticity.

From Equation 14 we find that if $\vec{\Omega}_{1}$ is zero, then $\vec{\Omega}$ is also zero. This implies that when fluid vorticity is zero, then solid vorticity is necessarily zero.

In the absence of suspended magnetic particles, $\mathrm{N}$ is zero and magnetization $\mathrm{M}$ is also zero, so Equation 20 is identically satisfied and Equation 15 reduces to

$$
\left(1+\lambda \frac{\partial}{\partial t}\right) \frac{D \vec{\Omega}_{1}}{D t}=v\left(1+\lambda_{0} \frac{\partial}{\partial t}\right) \nabla^{2} \vec{\Omega}_{1}+\left(1+\lambda \frac{\partial}{\partial t}\right)\left(\vec{\Omega}_{1} \cdot \nabla\right) \vec{q}
$$

This equation is vorticity transport equation. The last term on the right hand side of Equation 22 represents the rate at which $\vec{\Omega}_{1}$ varies for a given particle, when the vortex lines move with the fluid (the strengths of the vortices remaining constant) and the rate of change of vorticity, which varies for a given particle due to stress relaxation time. The first term represents the rate of dissipation of vorticity through friction (resistance) and rate of change of vorticity due to strain retardation time.

\section{Two-Dimensional Case}

Here we consider the two-dimensional case:

$$
\text { Let } \vec{q}_{d}=q_{d_{x}}(x, y) \hat{i}+q_{d_{y}}(x, y) \hat{j}, \vec{q}=q_{x}(x, y) \hat{i}+q_{y}(x, y) \hat{j},
$$

where components $q_{d_{x}}, q_{d_{y}}$ and $q_{x}, q_{y}$ are functions of $x, y$ and $t$, then

$$
\vec{\Omega}=\Omega_{z} \hat{k}, \vec{\Omega}_{1}=\Omega_{1 z} \hat{k} .
$$

In two-dimensional case, Equation 21 becomes

$$
\frac{D \Omega_{z}}{D t}=\frac{\mu_{0} \varepsilon}{m N}\left(\frac{\partial M}{\partial T}\right)\left(\frac{\partial T}{\partial x} \frac{\partial H}{\partial y}-\frac{\partial H}{\partial x} \frac{\partial T}{\partial y}\right)+\frac{K}{m}\left(\Omega_{1 z}-\Omega_{z}\right) .
$$

Similarly, Equation 16 becomes

$$
\left(1+\lambda \frac{\partial}{\partial t}\right) \frac{D \Omega_{1 z}}{D t}=v \nabla^{2}\left(\Omega_{1 z}\right)+v \lambda_{0} \frac{\partial}{\partial t} \nabla^{2}\left(\Omega_{1 z}\right)+\frac{K N}{\rho}\left(\Omega_{z}-\Omega_{1 z}\right)+\frac{K N \lambda}{\rho} \frac{\partial}{\partial t}\left(\Omega_{z}-\Omega_{1 z}\right),
$$

Because it can be easily verified that

$$
(\vec{\Omega} . \nabla) \vec{q}_{d}=0 \text { and }\left(\vec{\Omega}_{1} . \nabla\right) \vec{q}=0 \text {. }
$$

The first term on the right hand side of Equation 26 is the change of fluid vorticity due to internal friction (resistance), the second term is the rate of change of fluid vorticity due to strain retardation time, the third term is change in fluid vorticity on account of exchange of vorticity between solid and liquid and the fourth term rate of change in fluid vorticity on account of exchange of vorticity between solid and liquid due to stress relaxation time. Equation 26 does not involve explicitly the term representing change of vorticity due to magnetic field gradient and/or temperature gradient. But Equation 25 shows that solid vorticity $\Omega_{z}$ depends on these factors. Hence, it follows that fluid vorticity is indirectly influenced by the temperature and the magnetic field gradient.

In the absence of magnetic particles, $\mathrm{N}$ is zero and magnetization $\mathrm{M}$ is also zero, so Equation 25 is identically satisfied and Equation 26 reduces to classical equation for the transport of fluid vorticity. If we consider a suspension of non-magnetic particles instead of magnetic fluid, then the corresponding equation for the transport of vorticity may be obtained by setting $M$ equal to zero in the equations governing the transport of vorticity in magnetic fluids. If the magnetization $\mathrm{M}$ of the magnetic particles is independent of temperature, then the first term in Equations 20 and 25 vanishes and so the equations governing the transport of vorticity in magnetic fluid become the same as those governing the transport of vorticity in non-magnetic suspensions.

If the temperature gradient $\nabla T$ vanishes or if the magnetic field gradient $\nabla H$ vanishes or if $\nabla T$ is parallel to $\nabla H$, then also the first term in Equations 20 and 25 vanishes. Thus, we see that in this case also the transport of vorticity in magnetic fluid is same as transport of vorticity in non-magnetic suspension. 


\section{References}

[1] Wagh, D.K. and Jawandhia, A. (1996). Transport of vorticity in magnetic fluid. Indian J. Pure Appl. Phys., 34: 338-340. Saffman, P. (1962). On the stability of a laminar flow of a dusty gas, J. Fluid Mech., 13: 120-128. Wagh, D.K. (1991). A mathematical model of magnetic fluid considered as two-phase system. Proc. Int. Symp. on Magnetic Fluids, held at REC Kurukshetra, India, during Sept. 21-23, 182.

[4] Yan, Y. and Koplik, J. (2009). Transport and sedimentation of suspended particles in inertial pressure-driven flow. Phys. Fluids, 21: 013301.

[5] Oldroyd, J.G. (1958). Non Newtonian effects in steady motion of some idealized elastico-viscous liquids. Proc. Roy. Soc. London, A245: 278-297.

[6] Toms, B.A. and Strawbridge, D.J. (1953). Elastic and viscous properties of dilute solutions of polymethyl methacrylate in organic liquids. Trans. Faraday Soc., 49: 1225-1232.

[7] Bhatia, P.K. and Steiner, J.M. (1972). Convective instability in a rotating viscoelastic fluid layer, Z. Angew. Math. Mech., 52: 321-324.

[8] Sharma, R.C. (1976). Effect of rotation on thermal instability of a viscoelastic fluid. Acta Physica Hung, 40: 11-17.

[9] Hamabata, H. and Namikawa, T. (1983). Thermoconvective waves in a viscoelastic liquid layer. J. Phys. Soc. Japan, 52: 90-93.

[10] Kumar, P., Mohan, H. and Singh, G.J. (2004). Rayleigh-Taylor instability of rotating oldroydian viscoelastic fluids in porous medium in presence of a variable magnetic field. Transport in Porous Media, 56: 199-208.

[11] El-Sayed, M.F. (2008). Onset of electroconvective instability of Oldroydian viscoelastic liquid layer in Brinkman porous medium. Archive of Applied Mathematics, 78(3): 211-224.

[12] Hoshoudy, G.A. (2011). Quantum effects on the Rayleigh-Taylor instability of viscoelastic plasma model through a porous medium. J. Modern Physics, 2(10): 1146-1155.

[13] Kumar, P. and Mohan, H. (2012). Double-diffusive convection in a viscoelastic fluid. Tamkang J. Mathematics, 43: 365-374.

[14] Kumar, P. and Mohan, H. (2012). Thermal instability of a heterogeneous Oldroydian viscoelastic fluid heated from below in porous medium. J. Theroretical Applied Mechanics, 50: 943-951.

[15] Le Roux, C. (2014). On flows of viscoelastic fluids of Oldroyd type with wall slip. J. of Mathematical Fluid Mechanics, 16(2): 335-350.

[16] El-Sayed, M.F., Haroun, M.H. and Mostapha, D.R. (2014). Electrohydrodynamic peristaltic flow of a dielectric Oldroydian viscoelastic fluid in a flexible channel with heat transfer. J. Appl. Mech. Tech. Physics, 55(4): 565-577.

[17] Kumar, V. and Kumar, P. (2015). Thermosolutal convection in a viscoelastic dusty fluid with Hall currents in porous medium. Egyptian J. of Basic and Applied Sciences, 2(3): 22 1-228.

[18] Kumar, V., Kumar, P. and Awasthi, M.K. (2015). Hydrodynamic and Hydromagnetic triply diffusive convection in a viscoelastic fluid through porous medium. Special Topics \& Reviews in Porous Media: An International Journal, 6(3): 297-311.

[19] Rosensweig, R.E. (1997). Ferrohydrodynamics. Mineola, New York: Dover Publications, Inc. 